\section{Evolução histórica das bulas de medicamentos no Brasil}

\author{
Historical evolution of package inserts in Brazil
}

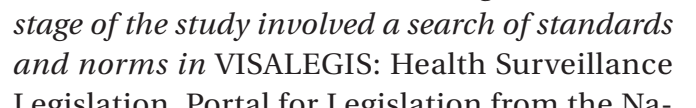
and norms in VISALEGIS: Health Surveillance
Legislation, Portal for Legislation from the Na-
tional Congressional Information System and Legislation, Portal for Legislation from the Na-
tional Congressional Information System and
the Health Legislation System. Package inserts
became an important vehicle for information in the country and underwent important regulatory changes in the latter half of the 20th century. 
tivo produzido pela indústria farmacêutica antes de sua comercialização. Esse processo se baseia tanto em informações presentes nos registros de medicamentos como naquelas encontradas em bancos de informações. O ponto chave é a análise da informação apresentada pelo detentor do registro e o confronto dessa informação com a literatura científica.

A forma e o conteúdo das bulas de medicamentos são legalmente determinados e acompanham a evolução da normatização do setor farmacêutico. Este artigo tem como objetivo analisar a evolução histórica dessa regulamentação legal das bulas de medicamentos no Brasil.

\section{Métodos}

As normas foram coletadas retrospectivamente a partir de 2005, período marcado pela publicação do Bulário Eletrônico da ANVISA (http://bulario. anvisa.gov.br, acessado em 08/Set/2007) e do 1 o Compêndio de Bula de Medicamentos (CBM), até a criação do Departamento Nacional de Saúde Pública (DNSP) em 1920. Seguiu-se a lógica de que a cada norma publicada, outro dispositivo era revogado.

A fonte inicial de coleta dos dados foi a $\mathrm{Co}$ leção Farmácia Brasileira - Portarias Sanitárias, editada pelo Ministério do Trabalho e Conselho Federal de Farmácia. Complementações foram feitas nos portais de pesquisa de legislação da ANVISA (VISALEGIS - Legislação em Vigilância Sanitária; http://e-legis.anvisa.gov.br, acessado em 08/Set/2007), do Ministério da Saúde (Saúde Legis; http://portal.saude.gov.br/saudelegis, acessado em 08/Set/2007) e do Senado Federal (Portal Legislação; http://portal.saude.gov.br/ saudelegis, acessado em 08/Set/2007).

Todos os resultados das buscas apresentavam o número e a data da norma, o órgão que a editou e o resumo do assunto ou ementa. Para aqueles que não apresentavam endereço eletrônico foram solicitadas cópias originais das edições do Diário Oficial da União à procuradoria da ANVISA, à biblioteca do Ministério da Saúde e, em último caso, ao Arquivo Nacional da Imprensa Nacional.

A legislação foi listada em ordem cronológica, em tabela classificando período de governo, estrutura institucional, tipo e número da norma legal, data da assinatura e da publicação oficial, área e assunto. Foi também classificada por áreas: organização de serviços, reunindo normas que tratam da estrutura institucional (ministérios, serviços, departamentos etc.) e das regras para indústria farmacêutica; registro, relativas à licença ou ao registro de medicamentos; me- dicamentos, que tratavam de diversos assuntos (classificação terapêutica, restrição de venda e uso etc.) relacionados a medicamentos e que afetavam as informações disponíveis nas bulas; rotulagem e bulas, com normas que citavam regras gerais para rotulagem e bulas; bulas, que reuniu a legislação específica de bulas de medicamentos; e farmácia de manipulação, incluindo normas sobre bulas para medicamentos manipulados.

\section{Resultados}

De 1953 a 2006, destacam-se quatro atos normativos sobre a estrutura e o conteúdo das bulas: a Portaria $n^{\circ} .49$, de 10 de agosto de 1959 5, do Serviço Nacional de Fiscalização da Medicina e Farmácia (SNFMF); a Portaria $n^{\circ}$. 65, de 28 de dezembro de 1984 6, da Secretaria Nacional de Vigilância Sanitária (SNVS); a Portaria $n^{\circ}$. 110, de 10 de março de 1997 7, da Secretaria de Vigilância Sanitária (SVS); e a Resolução da Diretoria Colegiada (RDC) $n^{\circ}$. 140, de 29 de maio de 2003 8, da ANVISA.

As normas específicas sobre os princípios ativos, do período de 1950 a 1973, continham as instruções que deveriam constar no ato do licenciamento do produto (antigo registro do medicamento). Observou-se que indicações terapêuticas, esquema posológico, restrição de venda, efeitos colaterais eram definidos para algumas substâncias, o que refletia em alterações nas bulas e rótulos daqueles medicamentos licenciados antes da publicação da norma. Além disso, notaram-se a padronização e a inclusão de frases de advertência.

Notou-se que, antes da criação do Ministério da Saúde, em 1953, a bula foi tratada em legislação não específica, a destacar o Decreto $n^{\circ}$. 20.377, de 8 de setembro de 1931 9, que regulamentou o exercício da profissão farmacêutica no Brasil. No capítulo sobre a Indústria Química e Farmacêutica, ao tratar da licença de especialidade farmacêutica, a bula é considerada pela primeira vez como um documento necessário para avaliação do pedido de licença. Em janeiro de 1946, o Decreto $n^{\circ}$. 20.39710 aprovou o Regulamento da Indústria Farmacêutica no Brasil, a ser aplicado pelo SNFMF. O capítulo Dos Produtos Químico-Farmacêuticos trata da licença de especialidades farmacêuticas e dos itens a constar nos rótulos e bulas.

O primeiro regulamento específico para as bulas, em que se define a ordem dos itens, ocorreu em 1959 com a Portaria $n^{\circ} .49$ 5. Essa norma regulamentou a apresentação e o exame de rótulos e textos de bulas, entretanto dispensou a sua 
obrigatoriedade quando as informações constassem na rotulagem. Além disso, determinou que a mesma não poderia conter informações diferentes daquelas apresentadas no ato da licença da especialidade farmacêutica.

Posteriormente, em 1977, o Decreto $n^{\circ} .79 .094$ 11, regulamentou a Lei $n^{\circ}$. 6.360/1976, e submeteu medicamentos e outros produtos e serviços à vigilância sanitária; ambas as normas estão em vigor. No Título X, Da Rotulagem e Publicidade desse Decreto, descrevem-se regras para bulas, rótulos, etiquetas e materiais impressos dos produtos de interesse sanitário. A bula, além de outros materiais impressos, deveriam ter dimensões necessárias à fácil leitura visual, observado o limite mínimo de $1 \mathrm{~mm}$ de tamanho de letra. Além disso, as contra-indicações, precauções e efeitos colaterais deveriam ser impressos em tipos maiores dos que os utilizados nas demais indicações e em linguagem acessível ao público. Foi a primeira a tratar do tamanho mínimo de letra e da necessidade de linguagem acessível ao leigo.

A Portaria $n^{\circ} .65$, da SNVS, instituiu o roteiro para texto de bula (modelo padrão de bula). A Divisão de Medicamentos (DIMED) ficou responsável pelos estudos dos textos, inicialmente por classe terapêutica e posteriormente produto a produto, determinando quais itens de informação obrigatória e, quando fosse o caso, quais textos padrões deveriam ser reproduzidos, o que na prática não ocorreu. Além disso, a portaria dividiu as informações em identificação do produto, informação ao paciente, informação técnica e dizeres legais.

Em 1997, a SVS publicou a Portaria $n^{\circ} .1107$ mantendo essa estrutura, com a inclusão de outros itens obrigatórios. Seguindo a tendência da Portaria $n^{\circ} .65^{6}$, de 1984, a SVS se comprometeu a elaborar dizeres de bula para cada princípio ativo ou associação, de acordo com a respectiva classe terapêutica. A inovação foi a classificação das informações em mínimos essenciais e máximos essenciais. Nos primeiros, os itens não poderiam ser omitidos e as novas informações deveriam ser baseadas cientificamente. Os mínimos essenciais compreendiam toda informação ao paciente e os subitens da informação técnica: contra-indicações, advertências, interações medicamentosas, reações adversas, superdosagem, pacientes idosos. Os máximos essenciais se referiam às indicações e posologia, e não poderiam ser acrescentadas informações além daquela padronizada. Ficou a cargo da SVS publicar em Diário Oficial da União os mínimos e máximos essenciais. Os demais itens, que dependiam das especificações do produto, caberiam à empresa elaborá-los. Publicações dos mínimos e máxi- mos essenciais foram efetivadas apenas para os medicamentos bromocriptina e terfenadina.

A ANVISA, criada como uma autarquia especial com independência administrativa, estabilidade de seus dirigentes e autonomia financeira ${ }^{12}$, substituiu a SVS após a publicação do Regimento Interno, pela Diretoria Colegiada. A Portaria $n^{\circ}$. 593, de 25 de agosto de 2000, definiu a sua estrutura organizacional 13, marcando o fim da SVS. Com a tarefa de revisar o arcabouço legal, a ANVISA editou em 2003 o conjunto de normas de registro de medicamentos.

Nesse contexto a legislação específica de bulas foi reformulada e publicada como Resolução $R D C n^{\circ} .140$, de 29 de maio de 2003 8, que dividiu as bulas em duas: bula para o paciente (identificação do medicamento, informações ao paciente, dizeres legais) e bula para o profissional de saúde (identificação do medicamento, informações técnicas aos profissionais de saúde, dizeres legais). Na informação ao paciente observa-se o reforço para a utilização de linguagem acessível ao organizar o texto em forma de perguntas e respostas, o que estabelece um "diálogo" entre a bula e o leitor. Outros pontos a serem destacados, ao comparar com a legislação anterior são: criação da lista de medicamentos padrão para texto de bula (para harmonizar o conteúdo das bulas nacionais, a partir da criação de um texto padrão para cada princípio ativo); aumento do tamanho mínimo de letra de $1 \mathrm{~mm}$ para 1,5mm; adoção da classificação de risco para gestantes; utilização da Classificação Internacional de Doenças (CID-10) ao se referir a sinais, sintomas e doenças; atualização das informações comparando com bulas internacionais; apresentação dos resultados dos estudos de eficácia; inclusão da descrição das características organolépticas do medicamento (para auxiliar na detecção de medicamentos falsificados ou com alterações); envio da bula eletronicamente pelo Sistema de Gerenciamento Eletrônico de Bulas (E-bulas; http://e-bulas.anvisa.gov.br) e publicação oficial dos textos no Bulário Eletrônico da ANVISA e no CBM. A Tabela 1 compara os itens exigidos ao longo da publicação de normas sanitárias consideradas impactantes.

Em 2004, a Resolução RDC $n^{\circ} .6814$ prorrogou o prazo final de envio das primeiras bulas padrões para 23 de abril, justificado pelo congestionamento de envio de bulas eletrônicas ao E-bulas. Durante a avaliação dos textos, a equipe do Projeto Bulas encontrou uma série de erros na configuração eletrônica das bulas, explicadas pela inexperiência das indústrias com a tecnologia adotada para o envio de arquivos. Assim, determinou-se em 2 de julho o reenvio de $90 \%$ das bulas encontradas na base de dados. Encer- 


\begin{tabular}{|c|c|c|c|c|c|c|}
\hline Itens obrigatórios & $\begin{array}{c}\text { Decreto } n^{\circ} \text {. } \\
20.397 / 1946 \text { * }\end{array}$ & $\begin{array}{l}\text { Portaria } n^{\circ} \text {. } \\
49 / 1959 \text { ** }\end{array}$ & 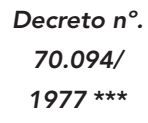 & $\begin{array}{c}\text { Portaria } n^{\circ} . \\
65 / 1984 \#\end{array}$ & $\begin{array}{l}\text { Portaria n } \\
\text { 110/1997 \#\# }\end{array}$ & $\begin{array}{c}\text { Resolução } \\
\text { RDC no. } \\
\text { 140/2003 \#\#\# }\end{array}$ \\
\hline \multicolumn{7}{|l|}{ Características específicas do medicamento } \\
\hline Nome comercial & $x$ & $x$ & $x$ & $x$ & $x$ & $x$ \\
\hline \multicolumn{7}{|l|}{ Nome técnico do componente ativo ou } \\
\hline denominação genérica do princípio ativo & $x$ & $x$ & $x$ & $x$ & $x$ & $x$ \\
\hline Nomenclatura oficial botânica & & & & & & $x$ \\
\hline Fórmula & $x$ & $x$ & $x$ & $x$ & $x$ & $x$ \\
\hline Peso, volume líquido ou quantidade de unidades & & & $x$ & $x$ & $x$ & $x$ \\
\hline Via de administração & & & $x$ & $x$ & $x$ & $x$ \\
\hline Formas farmacêuticas e apresentações & & & & $x$ & $x$ & $x$ \\
\hline Uso pediátrico ou adulto & & & & $x$ & $x$ & $x$ \\
\hline \multicolumn{7}{|l|}{ Ação e indicação do medicamento } \\
\hline Indicações terapêuticas & $x$ & $x$ & $x$ & $x$ & $x$ & $x$ \\
\hline Ação do medicamento & & & & $x$ & $x$ & $x$ \\
\hline Características químicas e farmacológicas & & & & & $x$ & $x$ \\
\hline Resultados de eficácia & & & & & & $x$ \\
\hline \multicolumn{7}{|l|}{ Riscos do uso do medicamento } \\
\hline Contra-indicações & & & $x$ & $x$ & $x$ & $x$ \\
\hline Precauções e advertências & & & & $x$ & $x$ & $x$ \\
\hline Interações medicamentosas & & & & $x$ & $x$ & $x$ \\
\hline Reações adversas & & & & $x$ & $x$ & $x$ \\
\hline Uso na gravidez e lactação & & & & $x$ & $x$ & $x$ \\
\hline \multicolumn{7}{|l|}{ Categoria de risco de fármacos destinados às } \\
\hline mulheres grávidas (frase de alerta) & & & & & & $x$ \\
\hline Riscos da automedicação & & & & $x$ & $x$ & \\
\hline Uso em pacientes idosos & & & & & $x$ & $x$ \\
\hline Restrição por faixa etária (frase de alerta) & & & & & & $x$ \\
\hline \multicolumn{7}{|l|}{ Orientações quanto ao uso do medicamento } \\
\hline Modo de usar ou cuidados de administração & $x$ & $x$ & $x$ & $x$ & $x$ & $x$ \\
\hline Modo de preparo & & & $x$ & $x$ & $x$ & $x$ \\
\hline Dose ou posologia & $x$ & & $x$ & $x$ & $x$ & $x$ \\
\hline Prazo de validade & & $x$ & $x$ & $x$ & $x$ & \\
\hline Prazo de validade após abertura da embalagem & & & & $x$ & $x$ & $x$ \\
\hline \multicolumn{7}{|l|}{ Cuidados de armazenamento antes e depois } \\
\hline da abertura & & & & $x$ & $x$ & $x$ \\
\hline Condutas em caso de superdosagem & & & & $x$ & $x$ & $x$ \\
\hline Conduta em caso de dose omitida & & & & & & $x$ \\
\hline \multicolumn{7}{|l|}{ Aspecto físico e características organolépticas } \\
\hline do medicamento & & & & & & $x$ \\
\hline
\end{tabular}

(continua)

rada a análise da linguagem, a Consulta Pública $n^{\circ}$. 51, de 11 de agosto de 2004 15, disponibilizou as bulas padrões para contribuições da sociedade científica, empresas farmacêuticas, centros de informação sobre medicamentos e profissionais de saúde. Além disso, foi publicada para cada bula padrão uma lista de medicamentos genéricos e uma lista de outros medicamentos com o mesmo princípio ativo (similares). Os detentores de registro de medicamentos genéricos e/ou similares deveriam conferir se seus medicamentos estavam devidamente relacionados aos padrões 


\begin{tabular}{|c|c|c|c|c|c|c|}
\hline Itens obrigatórios & $\begin{array}{c}\text { Decreto } n^{\circ} \text {. } \\
20.397 / 1946 \text { * }\end{array}$ & $\begin{array}{l}\text { Portaria } n^{\circ} . \\
49 / 1959 * *\end{array}$ & $\begin{array}{c}\text { Decreto } n^{\circ} \text {. } \\
70.094 / \\
1977^{\star \star \star}\end{array}$ & $\begin{array}{c}\text { Portaria } n^{\circ} . \\
65 / 1984 \text { \# }\end{array}$ & $\begin{array}{l}\text { Portaria } n^{\circ} . \\
110 / 1997 \text { \#\# }\end{array}$ & $\begin{array}{c}\text { Resolução } \\
\text { RDC n. } \\
\text { 140/2003 \#\#\# }\end{array}$ \\
\hline \multicolumn{7}{|l|}{ Dizeres legais } \\
\hline Restrição de venda (sob prescrição médica) & & $x$ & $x$ & $x$ & $x$ & $x$ \\
\hline Número da licença ou registro & & $x$ & $\mathrm{x}$ & $\mathrm{x}$ & $\mathrm{x}$ & $\mathrm{x}$ \\
\hline Nome do farmacêutico responsável & & $x$ & $x$ & $x$ & $x$ & $x$ \\
\hline Dados do farmacêutico responsável & & & $\mathrm{x}$ & $\mathrm{x}$ & $\mathrm{x}$ & $\mathrm{x}$ \\
\hline Local de fabricação & & $x$ & $x$ & $x$ & $x$ & $x$ \\
\hline Dados do fabricante & & & $x$ & $x$ & $x$ & $x$ \\
\hline Telefone de atendimento ao consumidor & & & & & & $\mathrm{x}$ \\
\hline \multicolumn{7}{|l|}{ * Brasil 10; } \\
\hline \multicolumn{7}{|c|}{ ** Serviço Nacional de Fiscalização da Medicina e Farmácia 5; } \\
\hline \multicolumn{7}{|l|}{ 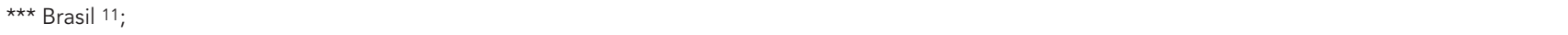 } \\
\hline \multicolumn{7}{|l|}{ \# Secretaria Nacional de Vigilância Sanitária 6; } \\
\hline \multicolumn{7}{|l|}{ \#\# Secretaria de Vigilância Sanitária 7; } \\
\hline Agência Nacional de Vigilância San & & & & & & \\
\hline
\end{tabular}

para texto de bula. Devido ao grande número de contribuições, a Resolução RDC $n^{\circ}$. 209, de 31 de agosto de 2004 16, prorrogou por mais trinta dias o prazo para envio das críticas e sugestões. Assim, as bulas padrões ficaram durante cinqüenta dias disponíveis no E-bulas para Consulta Pública.

Em 17 de maio de 2005, com a publicação da Resolução RDC $n^{\circ} .126$ 17, o Bulário Eletrônico da ANVISA e o CBM foram publicados. A partir dessa data as empresas com as bulas divulgadas nessas publicações liberariam no mercado as bulas atualizadas em dois formatos: bula para pacientes e bula para profissionais de saúde. A resolução reafirma que as bulas padrões deveriam estar contidas nas embalagens dos lotes a serem fabricados até o máximo de 180 dias. Além disso, a Resolução RDC $n^{\circ} .12617$ aprovou as Normas Relativas ao Livre Licenciamento do CBM e do Bulário Eletrônico da ANVISA. Assim, estabeleceram-se regras para aqueles que quisessem formular CBM.

Na 1ạ edição do CBM, de 2 mil exemplares, constam 572 bulas de medicamentos, que correspondem a 288 princípios-ativos alopáticos e aproximadamente 270 de imunobiológicos distribuídos em dois volumes, em um total de 4 mil páginas impressas. O Bulário Eletrônico da ANVISA foi concebido para ser um banco de dados acessível via Internet aos profissionais de saúde e à população, inclusive portadores de deficiência visual, contendo as informações dos textos de bula dos medicamentos registrados e comercializados no Brasil. Ao visar ao uso e à prescrição racional de medicamentos, o portal traz ainda matérias sobre educação em saúde, notícias relacionadas à atualização das bulas, a legislação em vigor sobre o assunto, perguntas freqüentes e também outros endereços eletrônicos de interesse na área de saúde.

\section{Discussão}

Desde 1931, a bula é exigida como documento sanitário no pedido de licença e considerada um item obrigatório na concessão do registro. Há muito é reconhecida por ter se tornado um meio de informação importante sobre os medicamentos, bem como por sua influência no consumo 18 . As informações no texto não poderiam, e não podem, diferir daquelas que a empresa comprovou no ato do registro. Devido à intrínseca relação entre o registro e a bula, a normatização de ambas era alterada praticamente ao mesmo tempo. Assim, à medida que as exigências para garantir eficácia, segurança e qualidade dos medicamentos aumentavam, os itens obrigatórios das bulas cresciam, sejam na melhor descrição e definição como na criação de novos itens de informação.

A linguagem utilizada sempre representou grande dificuldade para o leigo. O leitor se depara com uma escrita técnica que pouco ou nada lhe “comunica”, sendo considerada esta uma falha histórica na normatização e fiscalização dos órgãos governamentais responsáveis 3 . No entanto, apenas em 1977, a necessidade de utilizar uma linguagem acessível nas bulas veio a ser mencionada na regulamentação, e somente em 2004 foram realizadas análises da linguagem utilizada nas bulas ao paciente. Mesmo assim, permane- 
cem muitos vícios de linguagem e costumes a serem ultrapassados.

Considerada como a principal fonte de informação aos pacientes ${ }^{4}$, a bula assume um papel fundamental na promoção do uso racional de medicamentos. Ao alertar sobre os riscos da automedicação, da interrupção do tratamento, bem como quanto à necessidade da prescrição, no tocante à dispensação de medicamentos tarjados, está consoante ao processo educativo dos usuários, um dos pontos que merece ênfase segundo os propósitos da Política Nacional de Medicamentos 19.

A bula pode e deve ser um instrumento para a cidadania, pois empodera o indivíduo ao informá-lo sobre o uso do medicamento e, ao fazê-lo, contribui como instrumento para que a relação entre médico-paciente seja alterada pela capacidade de questionamento do paciente 20 . Por sua vez, o profissional deverá conhecer mais profundamente o medicamento, de tal forma a convencer que o tratamento farmacológico trará naquele caso mais benefícios que riscos. Entretanto, deve-se mencionar que o número de bulas publicadas no Bulário Eletrônico da ANVISA e no CBM ainda é pequeno.

No campo da vigilância sanitária, desde a criação do SNFMF se observam tentativas na padronização das informações contidas em medicamentos com os mesmos princípios ativos. Os instrumentos normativos específicos para classes terapêuticas ou substâncias ativas publicados entre 1950 e 1972, demonstram essa tendência. Entretanto, é importante destacar que a lista de medicamentos padrão para texto de bula não é atualizada desde 2003, apesar do Ministério da Saúde determinar como meta, no Plano Nacional de Saúde 21, disponibilizar pela Internet $11 \mathrm{mil}$ apresentações de medicamentos até 2007. Após o lançamento do CBM e do Bulário Eletrônico da ANVISA em 2005, pouco movimento efetivo foi observado para implementar a nova estrutura de bula em todos os medicamentos comercializados no Brasil. Até o momento não foram enviadas eletronicamente as bulas de medicamentos genéricos e similares. Conseqüentemente, o trabalho de padronização da informação fica prejudicado e o impacto sobre a informação de medicamentos, reduzido. Assim, a legislação, o E-bulas, o Bulário Eletrônico da ANVISA e o CBM precisam ser revisados, pois representam um poderoso instrumento de disseminação de informação sobre medicamentos.

Outros pontos a considerar, o Bulário Eletrônico da ANVISA disponibiliza apenas a última bula atualizada no E-bulas e aprovada pela equipe do Projeto Bulas, permitindo um vácuo entre a publicação da bula e da comercialização do medicamento com o novo modelo. O CBM, por ser volumoso e apresentar bulas de apenas alguns medicamentos, não foi distribuído em grande escala; foram editados 2 mil exemplares. Sendo assim, o conceito de livro impresso deve ser revisto se o objetivo é aumentar o alcance da informação.

Finalmente, é inegável a evolução no cuidado com que a legislação trata as bulas de medicamentos nesse período analisado. Entretanto, desde maio de 2005, após a publicação das bulas padrões, não foram eleitos novos "medicamentos padrão para texto de bula" nem foram publicadas as bulas de medicamentos genéricos e similares. Isso pode prejudicar consideravelmente a implantação do novo paradigma, sendo alto o risco o Projeto Bulas ser desacreditado e a $R e$ solução $R D C n^{\circ} .140$ ser considerada mais uma tentativa de padronização, com roupagem contemporânea, mas sem continuidade.

\section{Resumo}

No Brasil, a bula é usada como fonte de informação sobre medicamentos. O estudo desenvolveu uma análise de sua evolução histórica, procurando observar a influência do desenvolvimento científico, da globalização da informação e das diferentes políticas de saúde. Procedeu-se uma revisão retrospectiva da legislação sanitária brasileira até 1920, ano da instituição do Departamento Nacional de Saúde Pública. A análise documental sobre a evolução da regulamentação sanitária no Brasil se iniciou pela Coleção Farmácia Brasileira - Portarias Sanitárias. Na segunda etapa as normas foram pesquisadas nos portais: VISALEGIS Legislação em Vigilância Sanitária, Portal de Legislação do Sistema de Informações do Congresso Nacio- nal e Sistema de Legislação da Saúde - Saúde Legis. $A$ bula tornou-se um importante veículo informativo no Brasil, passou por profundas transformações regulamentares na segunda metade do século XX. Entre 1946 e 2006 o número de itens obrigatórios aumentou e sua descrição aprofundou. Entretanto, a padronização da informação para os medicamentos com o mesmo princípio ativo não se efetivou, não obstante sua importância e apesar das diferentes iniciativas observadas nas normas legais.

Bulas de Medicamentos; Legislação Sanitária; Vigilância Sanitária 


\section{Colaboradores}

T. R. Caldeira, E. Perini e E. R. Z. Neves contribuíram substancialmente para a concepção e planejamento, ou análise e interpretação dos dados, e significativamente na elaboração do rascunho e na revisão crítica do conteúdo, além de participarem da aprovação da versão final do manuscrito.

\section{Referências}

1. Silva D. O português das bulas. O Dia Online 2007; 9 mai. http://odia.terra.com.br/educacao/htm/ geral_97439.asp (acessado em 08/Set/2007).

2. Houaiss A. Dicionário Houaiss da língua portuguesa. http://houaiss.uol.com.br (acessado em 20/Set/2006).

3. Gonçalves SA, Melo G, Tokarski MHL, BarbosaBranco A. Bulas de medicamentos como instrumento de informação técnico-científica. Rev Saúde Pública 2002; 36:33-9.

4. Silva T, Dal-Pizzol F, Bello CM, Mengue SS, Schenkel EP. Bulas de medicamentos e a informação adequada ao paciente. Rev Saúde Pública 2000; 34:184-9.

5. Serviço Nacional de Fiscalização da Medicina e Farmácia, Ministério da Saúde. Portaria nº. 49, de 10 de agosto de 1959. Regula a apresentação e o exame de rótulos e bulas de produtos farmacêuticos, produtos oficinais, dietéticos, cosméticos, de higiene, toucador. Diário Oficial da União 1959; 17 ago.

6. Secretaria Nacional de Vigilância Sanitária, Ministério da Saúde. Portaria no ${ }^{\circ}$ 65, de 28 de dezembro de 1984. Institui o roteiro para texto de bula de medicamentos (modelo padrão de bula). Diário Oficial da União 1984; $31 \mathrm{dez}$

7. Secretaria de Vigilância Sanitária, Ministério da Saúde. Portaria no ${ }^{\circ}$ 110, de 10 de março de 1997. Institui roteiro para texto de bula de medicamentos, cujos itens devem ser rigorosamente obedecidos quanto à ordem e conteúdo. Diário Oficial da União 1997; 8 mar.

8. Agência Nacional de Vigilância Sanitária, Ministério da Saúde. Resolução da Diretoria Colegiada no 140 , de 29 de maio de 2003. Institui roteiro para texto de bula de medicamentos quanto à forma $\mathrm{e}$ conteúdo e publica a lista de medicamentos padrão para texto de bula. Diário Oficial da União 2003; 24 set.

9. Brasil. Decreto $\mathrm{n}^{\circ}$. 20.377, de 8 de setembro de 1931. Aprova a regulamentação do exercício da profissão farmacêutica no Brasil. Coleção de Leis do Brasil 1931; $31 \mathrm{dez}$

10. Brasil. Decreto no ${ }^{\circ}$ 20.397, de 14 de janeiro de 1946 Aprova o Regulamento da Indústria Farmacêutica no Brasil. Diário Oficial da União 1946; 19 jan.

11. Brasil. Decreto no ${ }^{\circ}$ 79.094, de 5 de janeiro de 1977. Regulamenta a Lei no ${ }^{\circ}$ 6.360/76. Diário Oficial da União 1977; 7 jan.

12. Brasil. Lei no. 9.782, de 26 de janeiro de 1999. Define o Sistema Nacional de Vigilância Sanitária e cria a Agência Nacional de Vigilância Sanitária. Diário Oficial da União 1999; 27 jan.
13. Agência Nacional de Vigilância Sanitária, Ministério da Saúde. Portaria $n^{\circ}$. 593, de 25 de agosto de 2000. Aprova o Regimento Interno da Agência Nacional de Vigilância Sanitária. Diário Oficial da União 2000; 28 ago.

14. Agência Nacional de Vigilância Sanitária, Ministério da Saúde. Resolução da Diretoria Colegiada $\mathrm{n}^{\circ}$. 68, de 25 de março de 2004. Prorroga o prazo para envio das bulas determinado pela Resolução - RDC n 140/2003. Diário Oficial da União 2004; 29 mar.

15. Agência Nacional de Vigilância Sanitária, Ministério da Saúde. Consulta Pública n ${ }^{\circ}$. 51, de 11 de agosto de 2004. Abre o prazo de 20 dias para que sejam apresentadas críticas e/ou sugestões aos textos dos "Medicamentos Padrão para Texto de Bula", e ao anexo: normas relativas ao livre licenciamento do Compêndio de Bulas de Medicamentos e do Bulário Eletrônico. Diário Oficial da União 2004; 12 ago.

16. Agência Nacional de Vigilância Sanitária, Ministério da Saúde. Resolução da Diretoria Colegiada $\mathrm{n}^{\circ}$. 209, de 31 de agosto de 2004. Prorroga o prazo da Consulta Pública ${ }^{\circ}$. 51/2004 por mais 30 dias. Diário Oficial da União 2004; 1 set.

17. Agência Nacional de Vigilância Sanitária, Ministério da Saúde. Resolução da Diretoria Colegiada no 126, de 16 de maio de 2005. Dispõe sobre publicação da 1a Edição do Compêndio de Bulas de Medicamentos (CBM) e a disponibilização do Bulário Eletrônico da Anvisa. Diário Oficial da União 2005; 17 mai.

18. Barros JAC. Estratégias mercadológicas da indústria farmacêutica e o consumo de medicamentos. Rev Saúde Pública 1983; 17:377-86.

19. Ministério da Saúde. Portaria no ${ }^{\circ} .3 .916$, de 30 de outubro de 1998. Dispõe sobre a Política Nacional de Medicamentos. Diário Oficial da União 1998; 10 nov.

20. Arrais PSD, Barreto ML, Coelho HLL. Aspectos dos processos de prescrição e dispensação de medicamentos na percepção do paciente: estudo de base populacional em Fortaleza, Ceará, Brasil. Cad Saúde Pública 2007; 23:927-37.

21. Ministério da Saúde. Portaria no ${ }^{\circ}$ 2.607, de 10 de dezembro de 2004. Aprova a Política Nacional de Saúde. Diário Oficial da União 2004; 13 dez.

Recebido em 06/Jul/2007

Versão final reapresentada em 02/Out/2007

Aprovado em 30/Out/2007 